\title{
"Y camién te tengo que contar algo más". Análisis de dos relatos de una niña con Trastorno Específico del Lenguaje (TEL) ${ }^{1}$
}

\author{
"And I Also Need To Tell You Something Else". \\ Analysis of two Stories of a child with Specific \\ Language Impairment (SLI)
}

Lucía Marroco M.

Maestra y Lic. en Lingüística Universidad de la República

\section{RESUMEN}

En este trabajo se analizan aspectos morfosintácticos y discursivos de dos relatos producidos por una niña con Trastorno Específico del Lenguaje (TEL) en su consulta fonoaudiológica. Se analizan dos corpus. Uno de ellos es una narración espontánea introducida por la niña (Corpus 1), mientras que el otro es una renarración de un cuento leído por su fonoaudióloga con apoyo de las imágenes (Corpus 2). El objetivo es analizar cualitativamente aspectos morfosintácticos y discursivos de ambos relatos. Se intenta mostrar algunos aspectos que aparecen como característicos de las narraciones orales de los niños con TEL a partir de la descripción cualitativa de ambos corpus. Las dos narraciones muestran los desórdenes descritos en la bibliografía en lo que respecta al nivel morfosintáctico. Sin embargo, el análisis de aspectos discursivos muestra una diferencia entre los corpus, ya que se evidencia mayor desorganización y problemas de coherencia local en el Corpus 1 (narración espontánea de la niña). En el Corpus 2 se ve una secuencia temporal ordenada, con progresión temática acorde con lo leído, donde la dificultad mayor se observa en las estrategias de explicitación y recuperación de los referentes vinculados con la inclusión de secuencias dialógicas al interior de la narración.

Palabras clave: análisis de corpus, relatos, TEL.

\section{ABSTRACT}

This paper analyzes the morphosyntactic and discursive aspects of two stories produced by a girl with Specific Language Impairment. Two corpora were analyzed. The first one refers to a spontaneous narrative produced by the patient (Corpus 1 ), while the second one is a re-narration of a tale read by the speech therapist, with images support (Corpus 2). The aim of this study was to analyze qualitatively morphosyntactic and discursive aspects of both speeches. It attempts to show some of the characteristic features of the oral narratives produced by children with Specific Language Impairment from both corpora qualitative description. Both corpora showed the disorders described in the literature at the morphosyntactic level. Nevertheless, the analysis of the discursive aspects showed a difference between the corpora, as more disorganization and coherence problems are seen in Corpus 1 (spontaneous narrative of the child). Corpus 2 showed an organized temporal sequence with thematic progression according to the reading. In this corpus, more difficulty was observed in the clarification and recovery strategies of the references linked to the inclusion of dialogic sequences within the narrative.

Keywords: analysis of corpus, SLI, stories.

\footnotetext{
${ }^{1}$ Trabajo realizado en el marco del proyecto "Estudio de la organización morfosintáctica y discursiva de los relatos de pacientes con dificultades del lenguaje a partir del análisis lingüístico de corpus", CSIC, Programa Iniciación a la Investigación, Modalidad 1- 2013, ID 390.
} 


\section{Introducción}

Los niños que han sido diagnosticados con un Trastorno Específico del Lenguaje (TEL) presentan un grado de adquisición y desarrollo de la habilidad lingüística inferior a lo esperable para su edad. Generalmente este déficit se caracteriza, en sus inicios, por un retraso cronológico en la adquisición del lenguaje, un desarrollo lento de las 50 primeras palabras y la aparición tardía de las primeras combinaciones de palabras en sentido pleno. Con el tiempo, se evidencian importantes dificultades para la estructuración del lenguaje y conductas verbales atípicas que responden a una desviación respecto de los procesos habituales en el desarrollo del lenguaje (Codesio, 2006).

Los criterios más utilizados en la identificación de niños con TEL son los de exclusión e inclusión ${ }^{2}$. En el primer caso, se descarta la presencia de alteraciones. En este sentido, no formarían parte del grupo de niños con TEL aquellos que presentan como causa principal retraso mental, deficiencia auditiva, disturbios emocionales severos, anormalidades bucofonatorias y signos neurológicos claros. Los criterios de inclusión, en cambio, consideran los requisitos mínimos que un niño debe tener para ser diagnosticado con TEL. De esta manera, forman parte de esta población los niños que manifiestan un nivel cognitivo mínimo, superan un screening auditivo en frecuencias conversacionales, no presentan ninguna lesión que pudiera explicar el trastorno lingüístico o quienes no han sido

\footnotetext{
${ }^{2}$ Mendoza (2014) desarrolla además otros criterios utilizados en el diagnóstico de niños con TEL, a saber: especificidad, discrepancia y evolución.
}

diagnosticados dentro de algún cuadro del espectro autista (Mendoza, 2014).

En cuanto a las dificultades morfosintácticas de los niños con TEL, Aguado (1999) explica que los errores más frecuentes son la omisión y la sustitución de unos morfemas por otros. Según este autor, "a partir de estos fenómenos la sintaxis se reduce en complejidad y puede hacer el lenguaje ininteligible" (p. 100). En estudios realizados con niños de habla inglesa se ha constatado que los niños diagnosticados con TEL presentan, en su mayoría, dificultades en el uso de morfemas gramaticales ${ }^{3}$ (Bedore \& Leonard, 1998; Leonard, 2014). Morgan, Restrepo y Auza (2009) explican que el déficit gramatical en niños con TEL españoles es consistente con la descripción realizada para los hablantes de inglés, pero consideran que en nuestra lengua más que un marcador gramatical típico de TEL lo que existe es una alta variabilidad dentro de las dificultades gramaticales que dependen de los perfiles de cada niño.

Auza y Morgan (2013a) analizaron el empleo de la categoría artículos en niños con TEL y encontraron que estos niños hacen un uso de los artículos semejante al de los niños que tienen un menor nivel de lenguaje $y$, por lo general, menos edad. Los autores resaltan que las dificultades en el empleo de artículos definidos para los niños con TEL parecen estar ligadas (además de las perceptuales vinculadas con el poco peso fonético que presentan) a fallas para reconocer los rasgos semánticos por los

\footnotetext{
3 "Differences have been seen for many different morphemes; however, morphemes pertaining to finite verbs such as present third singular inflections, regular past inflections, copula forms, and auxiliary be forms appear to be the most consistently problematic morphemes for children with SLI in these studies" (Bedore \& Leonard, 1998).
} 
sustantivos a los que acompañan los artículos en las frases o sintagmas nominales. En cuanto al uso de los artículos indefinidos, sugieren que el problema es inherente a las características de la indefinitud en español y no a un problema solamente de los niños con TEL.

También Auza y Morgan (2013b) constataron que las preposiciones constituyen otra fuente de dificultad para la mayoría de los niños con TEL. Los errores más importantes se encontraron en las preposiciones $a$, en y con:

Si bien es cierto que $a$, en y con son monosilábicas, átonas y de corta duración, también es cierto que cada una tiene más de un valor funcional, lo que puede incrementar su dificultad para utilizarlas correctamente. Al ser preposiciones polisémicas, podría implicar que los niños invierten más tiempo en aprenderlas y dominar sus funciones, a diferencia de aquellas palabras que representen un significado más acotado (Auza y Morgan, 2013b).

En este estudio se destaca un predominio de las omisiones por sobre las sustituciones y comisiones en el uso de las preposiciones en los niños con TEL.

Por otra parte, para Vázquez (2013), algunos de los aspectos morfosintácticos característicos del TEL se relacionan con la producción de los sintagmas nominales y los sintagmas verbales. En el primer caso, las producciones agramaticales al interior del sintagma nominal estarían vinculadas con las dificultades para seleccionar de forma correcta los elementos que, eventualmente, pueden acompañar al núcleo "sustantivo", tales como los determinantes (por ejemplo, artículos definidos e indefinidos, cuantificadores, posesivos, demostrativos), adjetivos, oraciones de relativo. En este sentido, para los niños con TEL, cuanto más compleja es la estructura del sintagma nominal que pretenden producir, mayores son sus posibilidades de cometer errores gramaticales.

Las dificultades en relación con los sintagmas verbales, por su parte, tienen que ver con la complejidad de su núcleo. El verbo contiene, por un lado, categorías morfológicas de tiempo, modo, persona y número (en las formas verbales flexionadas o conjugadas) y, además, una especificación de la estructura argumental (con excepción de los verbos auxiliares y copulativos) y los papeles temáticos que corresponden a cada argumento $^{4}$ (Di Tullio, 2010; Hernanz y Brucart, 1980). La complejidad semántico-sintáctica de los verbos impondría a los niños con TEL una dificultad mayor para plasmar sintácticamente una idea (Vázquez, 2013).

En cuanto a los aspectos discursivos, se ha constatado que los niños con TEL presentan dificultades en sus habilidades narrativas (Acosta, Moreno y Axpe, 2013; Codesio, 2006; Coloma, 2013; Contreras González y Soriano Ferrer, 2004; Fresneda, 2004; Pavez, Coloma y Maggiolo, 2008; Pérez, 1997; Serra, 1997). Por este motivo, las actividades vinculadas con la narración se consideran un indicador poderoso de TEL. Lo anterior se debe a que la narrativa requiere la correcta sincronización de una serie de habilidades, tales como la secuenciación

\footnotetext{
${ }^{4}$ Por ejemplo: Pablo rompió el vidrio. "Romper" está conjugado en tercera persona del singular del pretérito perfecto del modo indicativo; sus argumentos: "Pablo", con papel temático de agente, y "el vidrio", con papel temático de paciente. Ejemplo tomado de Di Tullio (2010).
} 
de acontecimientos, la creación de un texto conexo a través del uso de marcadores lingüísticos explícitos y la comprensión de las relaciones causa-efecto (Fresneda, 2004).

Por su parte, en un trabajo de investigación, BefiLopes, Paiva Bento y Perissinoto (2008) dan cuenta de que todas las narraciones producidas por los niños con desarrollo normal del lenguaje (grupo control) eran coherentes y completas, mientras que solo la mitad de las producidas por el grupo de niños con TEL se configuraron de esa forma. Su trabajo corrobora el hecho de que los niños con TEL producen historias menos cohesivas, con muchos errores sintácticos, semánticos y morfológicos vinculados con los déficits lingüísticos que caracterizan a estos niños. Por esta razón, las autoras destacan la realización de actividades que involucren las habilidades narrativas durante la etapa diagnóstica y en el proceso terapéutico, ya que le permiten al fonoaudiólogo verificar las competencias lingüísticas, cognitivas y sociales de estos niños, además de obtener datos para la organización del proceso terapéutico.

Serra (1997) discute aspectos cognitivos y lingüísticos de las producciones de niños con TEL a partir del análisis de errores y omisiones que cometen. En su estudio da cuenta de dos tipos de dificultades exclusivas del grupo de niños con TEL en el marco de actividades que requieren ensamblar diversas oraciones (la descripción y la narración): las omisiones inapropiadas de núcleos verbales y nominales y las oraciones incomprensibles debido a fallas en la asignación de funciones gramaticales. Para este autor, en los niños con TEL pareciera manifestarse "una dificultad especial en representar, conceptuar y poner en forma lingüística todo aquello que es variable y todo lo que es dependiente o debe ser jerarquizado para ser expresable" (Serra, 1997).

Cualquier unidad discursiva se compone de elementos verbales organizados y relacionados entre sí de forma explícita e implícita. Se trata de un entramado que trasciende la suma de oraciones, ya que estas se relacionan coherentemente en torno a un tema (a través de diversos mecanismos de cohesión), conformando una unidad semánticopragmática (Casamiglia y Tusón, 2002) emitida en una situación concreta con una determinada intención comunicativa (Pavez et al., 2008). Desde el punto de vista discursivo, las narraciones permiten un análisis lingüístico que considera la organización del contenido (coherencia local y mecanismos de cohesión empleados; coherencia global) y la estructuración formal del discurso ${ }^{5}$ (Borzone de Manrique y Rosemberg, 2008; Karmiloff y KarmiloffSmith, 2005; Pavez et al., 2008).

En síntesis, de acuerdo con los antecedentes expuestos, los niños con TEL tienden a presentar dificultades en sus narraciones tanto en los aspectos vinculados con la organización formal de estas, como en los aspectos relacionados con la organización del contenido. Al mismo tiempo, este grupo de niños se caracteriza por evidenciar dificultades morfosintácticas relacionadas con el uso de artículos, pronombres clíticos, preposiciones y la omisión de núcleos nominales y verbales. Por último, producen un número significativamente menor de oraciones complejas, pero cometen mayor número de errores gramaticales (Befi-Lopes et al., 2008).

\footnotetext{
${ }^{5}$ Pavez et al. (2008) señalan que estos dos puntos de vista coexisten $y$ se interrelacionan $y$ se pueden distinguir metodológicamente para su estudio.
} 
Si se considera que las narraciones, en tanto prácticas discursivas, están constituidas por una secuencia de oraciones cuyos significados se relacionan coherentemente, dificultades morfosintácticas tales como la producción de secuencias agramaticales (por ejemplo, por omisión de verbos, omisión o sustitución de preposiciones, entre otras) alteran la estructuración sintáctica del discurso. Más aún, existen ciertos procedimientos sintácticos que funcionan como mecanismos de cohesión, tales como la pronominalización. Dificultades en el manejo de pronombres (por ejemplo, el uso de un clítico sin mencionar su antecedente) repercuten en la estructuración del discurso, ya que la sustitución por pronombres es uno de los mecanismos de cohesión que posibilita la continuidad temática del discurso.

Por lo anteriormente expuesto, el objetivo de este trabajo es analizar cualitativamente aspectos morfosintácticos y discursivos de dos relatos producidos por una niña con TEL de 9 años 10 meses, que asistió al Servicio de Fonoaudiología del Hospital Pereira Rossell en Montevideo, Uruguay, durante el año 2014. Se busca mostrar algunos aspectos que aparecen como característicos de las narraciones orales de los niños con TEL a partir de la descripción cualitativa de ambos corpus.

\section{Presentación del caso y de los procedimientos para obtener y analizar los corpus}

En este trabajo se analizan dos corpus de la niña “ $\mathrm{R}^{\text {”6 }}$ de 9 años 10 meses. La niña fue derivada al Servicio de Fonoaudiología del Hospital Pereira

\footnotetext{
${ }^{6}$ De aquí en más se mencionará a la niña con una letra ya que se preservará el anonimato y confidencialidad de la pequeña.
}

Rossell por la Cátedra de Neuropediatría de dicho hospital, donde fue evaluada por profesionales de diversas áreas (neuropediatra, psicólogo, fonoaudiólogo y maestro especializado en dificultades del aprendizaje) y diagnosticada con TEL. En los informes de cada uno de los profesionales se repara en la "desorganización discursiva" que presenta R. De ahí el interés por encontrar pistas lingüísticas que evidencien esa desorganización en dos relatos diferentes.

De acuerdo con el informe de R, elaborado por su fonoaudióloga tratante, su perfil de desarrollo lingüístico es compatible con un trastorno específico del lenguaje que compromete ambos dominios (expresivo y comprensivo). En el informe se detalla:

En las instancias dialógicas se observó un habla con prosodia particular, dificultades a nivel del ritmo (disfluencia oscilante, frecuentemente asociada con instancias emocionales) y persistencia de errores en la programación fonológica, así como en la riqueza y organización sintáctica. Presenta dificultades a nivel de procesamiento auditivo de la información lingüística que conduce a un deficiente manejo de las operaciones de conciencia fonológica. Las actividades de memoria e integración auditiva le resultaron particularmente exigentes. Se evidencian dificultades para establecer $y$ explicitar relaciones semánticas entre dos oraciones, así como para elaborar conceptos y proposiciones referidas a una situación sobre la base de inferencias, características que reflejan compromiso de las habilidades semánticas a nivel discursivo. 
La niña $R$ ingresó al sistema educativo formal (educación inicial) a los 4 años, presentando una buena adaptación. En el informe correspondiente a su evaluación neuropsicológica, su madre refiere que en primer año comenzaron los problemas: "La madre comenta que cuando no entienden lo que R expresa, esta se enoja y se aleja de la situación. Actualmente, en la escuela se distrae y se pelea con sus compañeros". Al momento de la recolección de sus narraciones, $\mathrm{R}$ cursaba tercer grado escolar.

El Corpus 1 es un fragmento de una narración espontánea producida e introducida por R. Se trata de una narración "sin desencadenante" (Shiro, 2007) en el marco de la conversación. Surgió de la necesidad e interés de la niña por contar una experiencia personal: la llegada de su perrito. Cabe destacar que R solía usar el espacio de la consulta fonoaudiológica para contar experiencias personales como episodios en el recreo de su escuela, juegos con sus amigos, situaciones en las que se lastimó, películas que había visto con su mamá y su hermana.

El Corpus 2, en tanto, corresponde a una renarración con apoyo de las láminas del cuento ${ }^{7}$ inmediatamente después de haber sido leído por su fonoaudióloga.

La elección de dos relatos diferentes responde al interés por observar y comparar cómo R secuencia temporalmente la información, teniendo en cuenta que el primer corpus demanda mayor grado de descontextualización al ser una narración espontánea de una experiencia personal no compartida por sus interlocutores. Por otra parte,

7 El cuento utilizado, Óscar tiene frío, del uruguayo Ricardo Alcántara, desglosado en los componentes de la gramática de las narraciones, se encuentra en el anexo. También se encuentra en el anexo el Corpus 1 completo. interesa analizar si se evidencia o no el mismo tipo de dificultades morfosintácticas en ambos relatos.

Las muestras fueron recolectadas mediante grabación y transcritas ortográficamente. Para recogerlas, se solicitó autorización al referente familiar de la niña por medio de un consentimiento informado, avalado por el Comité de Ética de la Facultad de Medicina de la Universidad de la República (Uruguay).

La recolección de los relatos se realizó en el marco de la consulta fonoaudiológica de la niña en el Servicio de Fonoaudiología del Hospital Pereira Rossell $^{8}$. La investigadora asistió a sus consultas y grabó en audio las sesiones en las que se le solicitaba como tarea específica renarrar cuentos o elaborar historias a partir de viñetas. La grabación de las sesiones (y no solamente una actividad en particular) permitió contar con relatos espontáneos de experiencias personales por parte de la niña.

Las transcripciones de las muestras fueron realizadas por la investigadora. La intervención de la fonoaudióloga se representa con " $F$ ", la de la niña aparece con " $\mathrm{R}$ " y con "I" la intervención de la investigadora. Los segmentos ininteligibles se representan con "XXX".

\footnotetext{
${ }^{8}$ Las muestras fueron recolectadas en el marco del proyecto "Estudio de la organización morfosintáctica y discursiva de los relatos de pacientes con dificultades del lenguaje a partir del análisis lingüístico de corpus", CSIC, Programa Iniciación a la Investigación, Modalidad 1- 2013, ID 390. Para llevarlo adelante, se acordó la colaboración de la docente Caligari y de sus pacientes. Si bien el proyecto se propuso, en un principio, analizar los relatos de pacientes con dificultades del lenguaje, al momento de comenzar su ejecución se homogeneizó la población y se recolectaron muestras de niños entre 6 y 10 años con diagnóstico de TEL. En este proyecto, la investigadora estuvo a cargo de la recolección de las muestras, transcripción y análisis, pero no presentó las actividades (por ejemplo, lectura de los cuentos) a los niños. Se acordó la grabación de las sesiones fonoaudiológicas y la inclusión de actividades específicas que permitieran elicitar narraciones.
} 
Para ordenar el análisis de ambos corpus, se resaltaron en negrita, subrayaron y destacaron en cursiva aquellas secuencias que son objeto de estudio y descripción. En el caso del Corpus 2, además de estas estrategias, se ubicó la renarración de la niña en un cuadro que contiene el esquema básico narrativo a partir de la gramática de las narraciones.

El análisis de cada corpus se separa en dos apartados. El primero aborda aspectos discursivos y el segundo aborda aspectos morfosintácticos. En cuanto a los primeros, interesa observar en ambos corpus cómo $\mathrm{R}$ secuencia temporalmente la información presentada para progresar temáticamente. Dado que el Corpus 1 es una narración de una experiencia personal, se atenderá especialmente a las relaciones temporales ligadas con la coherencia local. Como ha estudiado Labov (2001; 2011) en sus trabajos acerca de narraciones orales de experiencias personales, este tipo de relato se caracteriza por el recuento de hechos pasados a partir de la elaboración de oraciones temporalmente ordenadas según la sucesión de eventos vividos que se pretenden narrar.

En el caso del Corpus 2, dado que se trata de una renarración de un cuento infantil se atenderá, por un lado, a la estructuración formal que presenta siguiendo la gramática de las narraciones. En este sentido, interesa analizar si $\mathrm{R}$ estructura formalmente su renarración utilizando las categorías básicas. Por otro lado, y en relación con la organización del contenido, se atenderá a algunos mecanismos de cohesión empleados por $\mathrm{R}$ para avanzar temáticamente en su renarración. En particular, se analizarán los mecanismos utilizados por la niña para mantener y recuperar los referentes personajes del cuento.

Por último, en ambos corpus, el análisis de los aspectos morfosintácticos se desprende de la identificación de secuencias agramaticales.

\section{Análisis del caso: Corpus 1}

A continuación se presenta el análisis del caso, desglosado para cada corpus.

R: Y camién te tengo que contar algo más...

F: A ver contame.

R: Sí... bueno... iah! ¡sí! Teeeenemos un nuevo de la familia, que cambién es de la familia.

F: A ver que eso se está poniendo muy interesante...

R: Aier vino.

\section{F: ¿Quién vino?}

R: Es un bebé. ¡Un bebé! Mamá quería un perrito raza, raza, raza... eh... viste esos pelos que camién tienen rulos que camién...

F: Caniche.

R: Mamá tuvo porque lo sacó de la panza de la made porque $X X X$.

\section{F: ¿Porque qué?}

R: Mamá lo compró.

\section{F: ¿Dónde lo compró mamá al caniche?}

R: No lo sé pero lo compró, bueno io no sabía... Bueno en pimera clase sabía que camién iba a compar al caniche pero no sabía que camién ia aier... Así que aier, aier me ievó a la escuela, no sé qué hizo que camién vine de la escuela, salí porque no sé qué hice porque estuve en la escuela. 
F: ¿`Y tenés que saber lo que hizo mamá mientras vos estás en la escuela?

R: ¡No!

F: ¡Ah!

R: Así que salí de la escuela, mamá estaba con la mochila abieta, la mochila así ( $R$ hace gesto y cara de sorpresa, como si la mamá le mostrase algo que tiene dentro de su mochila). Entonces me dijo “ivení!”, me ievó del brazo y había un XXX.

\section{F: ¿Había un qué?}

R: Lo vamos en el taxi, mamá me ievó de la mano y nos vamos al taxi. Porque mamá buscó al taxi.

F: ¡De apuro! ¡Te llevó rapidito!

R: [Entonces vi al pero XXX con los ojos cerados $\mathrm{Y}$ listol $^{1}$. Lo vi, [mamá lo bajamos del taxi] ${ }^{2}$, [fuimos pa casal $^{3}$ luego tenía deberes igracias a Dios! Porque cooon es que como que camién mi maesta particular se fue el martes.

\section{Análisis de aspectos discursivos del Corpus 1}

El Corpus 1 es un fragmento de un corpus mayor. La selección de este fragmento se basa en el hecho de que conforma en sí mismo una unidad temática y comunicativa, a saber: la narración de una experiencia personal. Interesa analizar la coherencia local, es decir, las relaciones semánticas entre las oraciones que conforman el relato. En particular, se atenderá a las relaciones temporales: “(...) aquellas en que los significados de las oraciones aluden a eventos o estados que ocurren secuencialmente en el tiempo: unos primero (o antes) y otros después" (Pavez et al., 2008, p. 27).

En el marco de la conversación con su fonoaudióloga, $\mathrm{R}$ introduce el relato de la llegada de su perro. Se trata de la narración de una experiencia no compartida por su interlocutor. Por consiguiente, demanda mayor manejo de la descontextualización. Las intervenciones de la fonoaudióloga resaltadas en negrita reflejan una demanda de información (¿Quién vino? ¿Dónde lo compró mamá al caniche?) para ir colaborando en la presentación de las ideas, o bien, una inducción a la autocorrección o reformulación (¿Porque qué? ¿Había un qué?), con lo cual se hace evidente el papel de la fonoaudióloga para entramar con $\mathrm{R}$ su discurso narrativo, el cual toma una forma más dialógica que narrativa, necesaria para ayudar a $\mathrm{R}$ en la progresión temática.

La niña $R$ encuentra dificultades para expresar la simultaneidad de los hechos. Esta dificultad la obliga a verbalizar uno a uno los eventos: salió de su casa, fue a la escuela, su madre hizo cosas que R no sabe, porque no estuvo con ella. Presenta la sucesión de forma desordenada. Incluso, la conjugación del último verbo en primera persona del singular (hice) en lugar de tercera persona del singular para referirse a la mamá, también altera el significado de la construcción:

Así que aier, aier me ievó a la escuela, no sé qué hizo que camién vine de la escuela, salí porque no sé qué hice porque estuve en la escuela.

F: ¿Y tenés que saber lo que hizo mamá mientras vos estás en la escuela?

A pesar de las dificultades morfosintácticas de $R$ (que se analizarán en el próximo apartado) y que obligan a la fonoaudióloga a interrumpir el relato, $\mathrm{R}$ presenta el tema y a partir de él va progresando en la presentación de la información hasta el desenlace. En este punto, R evidencia dificultad para secuenciar 
temporalmente y de forma ordenada los acontecimientos vividos por ella: comienza por el final (marcado en el corpus con $\left.{ }^{1}\right]$ : ve al perro); continúa su relato con dos momentos que son temporalmente anteriores: bajarse del taxi $\left[{ }^{2}\right]$ e ir a la casa $\left.\left[{ }^{3}\right]\right)$. Inmediatamente después, incluye un nuevo tópico (resaltado en el corpus con negrita):

R: [Entonces vi al pero XXX con los ojos cerados y listo] ${ }^{1}$. Lo vi, [mamá lo bajamos del taxi] ${ }^{2}$, [fuimos pa casa] ${ }^{3}$ luego tenía deberes igracias a Dios! Porque cooon es que como que camién mi maesta particular se fue el martes.

El desorden en la presentación temporal de la información, en la secuenciación de las oraciones desajustan la coherencia local y, por ende, alteran la organización discursiva de su relato.

\section{Análisis de aspectos morfosintácticos del Corpus 1}

R utiliza "que camién" (que alterna en sus emisiones con "que cambién") en forma de muletilla. Ha integrado esta expresión a su habla de tal manera que aparece en prácticamente todas sus intervenciones.

El sintagma nominal "un nuevo de la familia" aparece sin su núcleo. Se trata de la omisión de núcleo nominal que no es recuperable por el contexto lingüístico. El sintagma nominal "un nuevo [integrante] de la familia" desempeña la función sintáctica de objeto directo del verbo "tenemos" empleado por R. El adjetivo "nuevo" modifica, en el sintagma de $\mathrm{R}$, a un núcleo que no está presente. Lo mismo sucede con el indefinido "un": determina un sustantivo ausente. "Nuevo", en esta secuencia, es adjetivo, no se recategoriza en sustantivo $y$, por lo tanto, no habilita un determinante indefinido no apocopado ("un"). De esta forma, en la secuencia "un nuevo de la familia" hay una elipsis inapropiada del núcleo nominal ${ }^{9}$.

En el caso del sintagma nominal "esos pelos que camién tienen rulos que camién", si bien "esos pelos que tienen rulos" no constituye un sintagma agramatical en la medida que "pelos" es el núcleo y la oración de relativo "que tienen rulos" modificaría a ese núcleo, $\mathrm{R}$ introduce ese sintagma en un momento de su relato en el cual pretende identificar al referente sobre el cual versará la narración de su llegada, a saber: el perro. Por lo tanto, presentar el referente (perro) y a partir de él una característica que lo defina (que tiene pelos con rulos) haría más aceptable y ajustado su discurso.

Respecto de la "agramaticalidad", Bosque y Gutiérrez-Rexach (2009) explican que los hablantes de una lengua, que no presentan patología, rechazan secuencias tales como "durante que te estuve esperando", dado que su sistema gramatical no permite que se combinen las propiedades gramaticales de esas palabras. Se produce un

\footnotetext{
${ }^{9}$ Di Tullio y Malcuori (2012) señalan que "la frontera entre los adjetivos y los sustantivos es muy lábil en español. Son muchos los adjetivos que tienen usos nominales (...) Por conversión, se forman nuevos sustantivos a partir de adjetivos" (p. 200). Esta situación podría generar dudas respecto de la clase gramatical (sustantivo o adjetivo) de algunas palabras en ciertos contextos. Las autoras explican que esta situación no debería traer aparejada la confusión entre la "conversión" y la "elipsis" habilitada por el sistema gramatical. Por ejemplo, en "Te traje el pantalón gris, pero me olvidé del negro", negro mantiene su condición de adjetivo (no hubo reconversión a sustantivo), ya que se interpreta como un núcleo elidido (el $\varnothing$ negro). Además, debe tenerse en cuenta que el artículo definido no cambia si hay un elemento nulo, pero en el indefinido no se produce la apócope de la última vocal, lo mismo que sucede con otros cuantificadores: \{uno-algunoninguno-cualquiera $\} \varnothing$ negro.
} 
"choque", un conflicto de propiedades gramaticales. Pues bien, en el caso de los niños con trastorno en el componente morfosintáctico, ese choque es constante, situación que obliga a los interlocutores a reinterpretar sus producciones y al fonoaudiólogo a colaborar en el reajuste del sistema gramatical. Al mismo tiempo que las secuencias agramaticales generan desconcierto en los interlocutores, van gestando una autopercepción negativa en los niños que padecen TEL, coartando, en muchos de ellos, la posibilidad de interactuar con su entorno.

En la intervención que se transcribe a continuación se pone de manifiesto la dificultad de $\mathrm{R}$ para encontrar el verbo adecuado a la idea que desea transmitir, verbo que puede desplegar luego de la pregunta de la fonoaudióloga. En tanto $\mathrm{R}$ no encuentra los verbos que más se ajustan a la información que pretende ofrecer, despliega frases animadas y mágicas (la madre de R saca al perrito de la panza de la perra):

Mamá tuvo porque lo sacó de la panza de la made porque $X X X$.

\section{F: ¿Porque qué?}

R: Mamá lo compró.

El fragmento que se analiza a continuación muestra cómo $\mathrm{R}$ acompaña su relato de recursos gestuales tales como el señalamiento de una mochila abierta. La inclusión de este tipo de recurso no facilitó la comprensión del relato por parte de sus interlocutores (la fonoaudióloga y la investigadora), ya que ambas entendieron que, dada la euforia y alegría con la que $\mathrm{R}$ representaba la mochila abierta, era esperable que estuviera el perrito adentro. Sin embargo, luego de reordenar con ella los acontecimientos (al final del corpus que se encuentra en el anexo) queda claro que $\mathrm{R}$ vio al perro por primera vez al llegar a su casa:

R: Así que salí de la escuela, mamá estaba con la mochila abieta, la mochila así ( $R$ hace gesto $y$ cara de sorpresa, como si la mamá le mostrase algo que tiene dentro de su mochila). Entonces me dijo "ivení!", me ievó del brazo y había un $X X X$.

\section{F: ¿Había un qué?}

R: Lo vamos en el taxi, mamá me ievó de la mano y nos vamos al taxi. Porque mamá buscó al taxi.

Podría afirmarse también que la duda acerca de si estaba o no el perro adentro de la mochila de su madre podría anularse si $\mathrm{R}$ no encontrara dificultades para expresar la finalidad: la mamá fue a buscarla en taxi y la llevó muy rápido para llegar a la casa y mostrarle al perrito.

Por último, R sustituye la preposición "en" por "a" en: nos vamos al taxi (nos vamos en taxi).

\section{Análisis del caso: Corpus 2, aspectos discursivos}

\section{Estructuración formal de la renarración a partir de} la gramática de las narraciones

A continuación, se desglosó en un cuadro la renarración producida por $\mathrm{R}$ luego de la lectura del cuento. En la columna de la izquierda aparecen las categorías básicas de la gramática de las narraciones subdivididas en otras categorías. En la columna derecha de la tabla se encuentra el relato de la niña. 
Tabla 1

Renarración de R del cuento Óscar tiene frío

\section{Presentación}

\begin{tabular}{ll}
\hline a) Marco & $\begin{array}{l}\text { Un pequeño oso que vi que cambién pasó todo el invieno en su cueva y cambién y cambién que y } \\
\text { cambién }\end{array}$ \\
\hline b) Ubicación espacial y temporal & vino la pimavera, se salió la cama con un salto y feliz bailó. \\
\hline c) Problema & \\
\hline
\end{tabular}

\section{Episodio 1}

$\begin{array}{ll}\text { a) Meta } & \text { Caminó, caminó, caminó, caminó hasta que se encontó a la liebe y le dijo: liebe, tengo mucho frío. } \\ & \text { ¿Por qué? iMe acabo sobe despetar! Quédate aquí y voy corriendo a buscate algo. Entonces un minuto, } \\ \text { volvió entonces, dijo: icúbete con esta sábana roja y cambién de lana! Te abiga y entarás en calor muy } \\ \text { ponto. }\end{array}$

Episodio 2

\begin{tabular}{ll}
\hline a) Meta & Caminó, caminó y caminó pero le pasó. Entonces se le encontó a una cigüeña. No, un pájaro, un pájaro y \\
& le digo: ¿Osito por qué tienes fío? \\
b) Intento para alcanzar la meta & Es que me acabo soooobe levantar en la cama y ia tengo fío. Quédate aquí que ia vengo volando. \\
& Entonces volvió, entonces le dio un chocolatito calentito y muy riquito.
\end{tabular}

c) Consecuencia

\section{Episodio 3}

a) Meta
b) Intento para alcanzar la meta
c) Consecuencia

C.

Se resuelve positiva o negativamente

el conflicto que generó la historia

\section{Caminó, con la manta, con el chocolito calentito y cambién ¿Qué era la ota?}

La ota era un paloma, no una... bueno ¿un zorro?

Era un zorro, entonces le hizo una fogata que cambién le quedó calentito todo el cuerpito. pero siguió teniendo fío.

\section{Final}

Ahora su ma, ahora su amiga le dio un fuerte abazo, un fuerte abazo, un fuerte abazo que casi lo ataba un fuete que ia se le calentó y ia nunca más tuvo fío, en los pelos ise le entró arie!,i más arie!, imás arie! Y camién ia se acostumbó.
La estructura formal del cuento infantil, derivada de la gramática de las narraciones, presenta tres categorías básicas: la presentación, el o los episodios y el final (Borzone de Manrique y Rosemberg, 2008; Karmiloff y Karmiloff-Smith, 2005; Pavez et al., 2008). En este caso, R logra estructurar formalmente el relato a partir de las categorías formales básicas y desde sus posibilidades y conocimientos. Presenta al personaje principal (un pequeño oso) y lo ubica espacial (en su cueva) y temporalmente (Ilegada de la primavera). De a poco, va introduciendo una serie de acontecimientos, de episodios orientados a una meta u objetivo (quitarse el frío) con un resultado (el oso continúa con frío). Introduce, en cada episodio, a los personajes secundarios que son los que intentan ayudar al oso; y por último, presenta el final de la 
historia con una resolución positiva, ya que el oso no tiene más frío.

De todos los elementos constitutivos de los episodios, $\mathrm{R}$ no explicita la consecuencia o resultado (el oso continúa con frío) que desencadena la colaboración de un personaje secundario. Lo hace solo en el último episodio. Sin embargo, R no vulnera el orden de los episodios y se esfuerza por sostener un eje temporal. $\mathrm{R}$ progresa temáticamente en el relato, aportando información nueva pero con dificultades para explicitar y mantener la referencia.

\section{Mecanismos de cohesión al interior de la renarración: el mantenimiento de la referencia}

La cohesión constituye una de las manifestaciones más importantes de la coherencia. Funciona como un conjunto de enlaces intratextuales para establecer las relaciones semánticas que necesita un texto para constituirse como unidad de significación (Casamiglia y Tusón, 2002).

Hay distintos mecanismos para mantener la referencia iniciada, por ejemplo, la repetición exacta o parcial del referente introducido. En el momento en el que se debilita la referencia (por la distancia entre una aparición y otra, o bien porque la memoria a corto plazo deja de funcionar) se repite el referente, con las mismas palabras o con variaciones.

Junto con la posibilidad de introducir los temas y mantenerlos con formas léxicas también se puede mantener la referencia a través de formas gramaticales, es decir, a partir de elementos lingüísticos que actúan con una función endofórica. Son piezas que reducen la redundancia sin dejar de señalar de qué se está hablando.
De acuerdo con lo anterior, en este apartado se realizará un análisis de los mecanismos empleados por $\mathrm{R}$ para mantener y recuperar los referentes personajes del cuento. Los criterios utilizados para analizar este fenómeno atienden al uso que hace $\mathrm{R}$ de las estrategias de repetición, de sustitución (por pronombres, posesivos, por una expresión semánticamente relacionada) y de omisión.

Así, R comienza su renarración mencionando al personaje principal en la presentación: “Un pequeño oso". Lo recupera recién en el episodio 2 bajo la forma de un diminutivo: “osito" (repetición parcial) en contexto de discurso directo, en la "voz" del pájaro. En el caso de la presentación no hay ambigüedad, ya que todas las formas verbales recuperan como sujeto al oso. Sin embargo, a medida que avanza en el relato, el personaje principal se va encontrando con otros personajes y se establece un diálogo con cada uno de ellos. Se podría afirmar que es el contexto de discurso directo el que ofrece mayores dificultades a $R$ para establecer la referencia obligada, ya que se necesita introducir en cada caso la "voz" de los personajes.

Episodio 1: Caminó, caminó, caminó, caminó hasta que se encontó a la liebe y le dijo: liebe, tengo mucho frío. ¿Por qué? ¡Me acabo sobe despetar! Quédate aquí y voy corriendo a buscate algo. Entonces un minuto, volvió entonces, dijo: icúbete con esta sábana roja y cambién de lana! Te abiga y entarás en calor muy ponto.

Episodio 2: Caminó, caminó y caminó pero le pasó. 
Cuando el oso se encuentra con la liebre, mantener el referente se vuelve necesario: marcar la intervención de cada personaje, explicitar quién toma la palabra, sobre todo al final del episodio 1 y comienzo del 2 , ya que en este pasaje $\mathrm{R}$ termina el episodio 1 en la "voz" de la liebre y comienza el episodio 2 con "su voz" de narradora haciendo referencia al oso, pero sin explicitar léxicamente los referentes. Probablemente, el ir y venir en las marcas dialógicas dentro de la narración sea una tarea ardua para los niños con compromiso en las habilidades referenciales y un desafío importante para abordar en la terapia fonoaudiológica.

A medida que avanza la renarración de $R$, no se evidencian mecanismos que permitan mantener la referencia del personaje principal a través de estrategias como la repetición (exacta o parcial), la sustitución por un pronombre personal (él) o por un elemento nominal semánticamente relacionado. S se evidencian procedimientos gramaticales vinculados a marcas verbales para hacer referencia a este y el uso de pronombres personales átonos (le, lo) que, siendo anafóricos, remiten a un antecedente. En este caso, en el relato de R, estos pronombres se van presentando sin la mención del elemento antecedente.

Por último, la elipsis como fenómeno de cohesión resulta un mecanismo óptimo para reducir la redundancia y evitar la excesiva repetición exacta de los referentes. En lenguas como el español, una forma de elipsis muy habitual atañe al sujeto, ya que al aparecer por primera vez en una oración, puede no figurar en otras oraciones del entramado discursivo hasta que se introduce un nuevo sujeto (Casamiglia y Tusón, 2002). Sin embargo, la elipsis de los elementos nominales que realiza $\mathrm{R}$ (personaje principal y personajes secundarios) en su renarración da cuenta de su dificultad en este aspecto. Obliga al interlocutor a inferir de qué personaje se trata. Para ejemplificar, se retoma el episodio 1 y se colocan entre paréntesis los referentes elididos:

(el oso) Caminó, caminó, caminó, caminó hasta que se encontó a la liebe y le dijo: liebe, tengo mucho frío. ¿Por qué? (la liebre) iMe acabo sobe despetar! (el oso) Quédate aquí y voy corriendo a buscate algo (la liebre). Entonces un minuto, volvió (la liebre) entonces, dijo: ¡cúbete con esta sábana roja y cambién de lana! Te abiga y entarás en calor muy ponto.

Episodio 3: (el oso) Caminó, caminó y caminó pero le pasó.

\section{Análisis de aspectos morfosintácticos del Corpus 2}

En relación con las dificultades a nivel morfosintáctico, en el Corpus 2 se observan:

a) Desórdenes en el régimen preposicional: "Me acabo sobe despertar", "me acabo soooobe levantar en la cama" (me acabo de levantar de la cama). En este punto, es de destacar que las secuencias agramaticales vinculadas con los sintagmas preposicionales producidos por $\mathrm{R}$ están relacionadas con la sustitución de las preposiciones y no con la omisión de estas (como se había hallado en la mayoría de los niños investigados en Auza. y Morgan (2013a).

b) Formas verbales distorsionadas, como "camentaba". Esta forma podría ser el resultado de la fusión de su muletilla "cambién" con el verbo "entraba", que es en definitiva lo que $\mathrm{R}$ 
quiere expresar: para ver si entraba en calor.

c) Omisión de verbos en contexto obligatorio: "Entonces (pasó) un minuto".

d) Flexión incorrecta de verbos debido al desorden en el nivel fonológico que presenta $R$ y que no se analizarán en este trabajo: "un pájaro y le digo" (dijo).

e) Error de concordancia de género entre el artículo y el sustantivo al interior de un sintagma nominal: "un paloma".

f) Omisión de un adverbio de negación necesario para mantener la coherencia local de su relato: “Caminó, caminó y caminó pero le pasó” (pero no se le pasó el frío).

g) Adición de pronombres no requeridos por el verbo: "se salió de la cama", "Entonces se le encontó a una cigüeña".

h) Uso de un adverbio con función deíctica ligado con su "aquí" de narradora (ahora) en lugar de un indicador espacio-temporal para introducir el final de la historia.

\section{Conclusiones}

A lo largo de este trabajo, se analizaron dos relatos diferentes de una niña de 9 años 10 meses diagnosticada con TEL, con la intención de encontrar en la descripción lingüística de sus relatos características morfosintácticas y discursivas. Esto con el fin de poder visualizar en dos corpus distintos algunos desórdenes descritos en la bibliografía en torno al tema como relevantes tanto a nivel morfosintáctico, como en el ámbito de la narrativa.

En primer lugar se evidencia, en ambos corpus de la niña, la dificultad que esta presenta a nivel morfosintáctico. Teniendo en cuenta los antecedentes presentados en la introducción, se constata que $\mathrm{R}$ evidencia dificultades vinculadas con los morfemas gramaticales (Aguado, 1999; Bedore \& Leonard, 1998), ya que se observan errores en la flexión verbal, así como en la concordancia al interior del sintagma nominal. También presenta dificultades en el uso de pronombres clíticos, preposiciones y problemas relacionados con la omisión de núcleos verbales y nominales. Los errores gramaticales más relevantes de $\mathrm{R}$ fueron estos últimos. En algunos casos, la agramaticalidad se debió a la ausencia del verbo en la oración o del núcleo sustantivo en el sintagma nominal. En reiteradas ocasiones, $\mathrm{R}$ evidenció dificultades en el manejo de las preposiciones vinculadas con la sustitución de unas por otras, más que a la omisión de esta clase de palabras, tal como encontraron Auza y Morgan (2013a) en su trabajo de investigación.

Si bien los desórdenes a nivel morfosintáctico son constantes en ambos corpus, el análisis de aspectos discursivos muestra una diferencia entre los dos relatos producidos por la niña. En primer lugar, se observa que su narración espontánea (Corpus 1) se construye colaborativamente con la fonoaudióloga: R necesita de su intervención para progresar temáticamente. Es por esta razón que el relato adopta una forma más dialógica que narrativa. A medida que avanza en este relato, se evidencia su dificultad para secuenciar temporalmente los eventos vividos por ella, tal como se mencionó en los antecedentes teóricos presentados en la introducción (Contreras González y Soriano Ferrer, 2004; Fresneda, 2004; Pavez et al., 2008). Al desorganizar la presentación de sus oraciones, $\mathrm{R}$ altera una de las relaciones de coherencia local 
característica de las narraciones, a saber: las relaciones temporales.

En cuanto a la renarración del cuento (Corpus 2) $\mathrm{R}$ estructura formalmente su relato según las categorías básicas de la gramática de las narraciones. Al mismo tiempo, organiza su contenido secuenciando correctamente los eventos y los presenta temporalmente sin errores. Este hecho podría reafirmar la inclusión de actividades de recontado de cuentos en la terapia fonoaudiológica (Befi-Lopes et al., 2008) como estrategia organizativa.

Por último, en la renarración del cuento se analizó uno de los mecanismos de cohesión, esto es, el mantenimiento y recuperación de los personajes del cuento. En él, se atendió al uso que hace $\mathrm{R}$ de las estrategias de repetición, sustitución y omisión. Se constata que la niña no recupera los personajes mediante la repetición léxica (total o parcial), la sustitución por pronombres o palabras semánticamente vinculadas. Por otro lado, se evidencia su dificultad en el uso de la elipsis como estrategia de omisión, ya que avanza en la presentación de las oraciones, manteniendo los referentes elididos cuando se necesita retomarlos y explicitarlos mediante alguno de los otros mecanismos. Podría afirmarse que la dificultad de $R$ está estrechamente vinculada con el discurso directo, el cual obliga a la explicitación de las voces (personajes) previa o posteriormente a la formulación del mensaje. En este sentido, la inclusión de secuencias dialógicas dentro de la narración probablemente implique una dificultad mayor para recuperar los referentes en los niños con TEL.

\section{Referencias}

Acosta V., Moreno, A., y Axpe, A. (2013). Análisis de las dificultades en el discurso narrativo en alumnado con Trastorno Específico del Lenguaje. Revista de Logopedia, Foniatría y Audiología, 33(4), 165-171. http://dx.doi.org/10.1016/j.rlfa.2013.07.004

Aguado, G. (1999). Trastorno específico del lenguaje. Retraso de lenguaje y disfasia. Málaga: Ediciones Aljibe.

Auza, A. y Morgan, G. (2013a). Uso de preposiciones en el recuento de una historia. Comparación de niños hispanohablantes con y sin trastorno de lenguaje. Revista Infancia y Aprendizaje, 36(1), 35-49. http://dx.doi.org/10.1174/021037013804826573

Auza, A. y Morgan, G. (2013b). El uso del artículo en niños hispanohablantes con Trastorno Específico del Lenguaje. Revista Chilena de Fonoaudiología, 12, 3-20. http://dx.doi.org/10.5354/0719-4692.2013.29513

Bedore, L. \& Leonard, L. (1998) Specific language Impairment and grammatical morphology: A discriminant function analysis. Journal of Speech, Language, and Hearing Research, 41(5), 1185-1192. http://dx.doi.org/10.1044/jslhr.4105.1185

Befi- Lopes, D., Paiva Bento, A., \& Perissinoto, J. (2008). Narração de histórias por crianças com distúrbio específico de linguagem. Pró-Fono Revista de Atualização Científica, 20 (2) 93-98. http://dx.doi.org/10.1590/s0104$\underline{56872008000200004}$

Borzone de Manrique, A. M. y Rosemberg, C. R. (2008). ¿Qué aprenden los niños cuando aprenden a hablar? El desarrollo cognitivo y lingüístico en los primeros años. Buenos Aires: Editorial Aique.

Bosque, I. y Gutiérrez-Rexach, J. (2009). Fundamentos de sintaxis formal. Madrid: Ediciones Akal.

Casamiglia, H. y Tusón, A. (2002). Las cosas del decir. Manual de análisis del discurso. Barcelona: Editorial Ariel.

Codesio, A. I. (2006). La estructura de los mensajes, su comprensión y su producción: nivel gramatical. En E. Garayzábal (Ed.), Lingüística clínica y logopedia (p. 231). Madrid: Antonio Machado Libros S.A. 
Coloma, C. J. (2013). Discurso narrativo en escolares de 10 básico con Trastorno Específico del Lenguaje (TEL). Revista Signos, 47(84), 3-20. http://dx.doi.org/10.4067/S0718$\underline{09342014000100001}$

Contreras González, M. C. y Soriano Ferrer, M. (2004). EI valor de la narrativa en la caracterización de los alumnos con dificultades de lenguaje. Revista de Logopedia, Foniatría y Audiología, 24(3), 119-125. http://dx.doi.org/10.1016/s0214-4603(04)75789-7

Di Tullio, A. (2010). Manual de gramática del español. Buenos Aires: Waldhuter Editores.

Di Tullio, A. y Malcuori, M. (2012). Gramática del español para maestros y profesores del Uruguay. Montevideo: Tradinco S. A.

Fresneda, M. D. (2004). La narrativa en el TEL. En E. Mendoza (Coord.), Trastorno Específico del Lenguaje (pp. 169). Madrid: Ediciones Pirámide.

Hernanz, M. L y Brucart, J. (1987). La sintaxis. Barcelona: Editorial Crítica.

Karmiloff, K y Karmiloff-Smith, A. (2005). Hacia el lenguaje. Del feto al adolescente. Madrid: Ediciones Morata S. L.

Labov, W. (2001). Uncovering the event structure of narrative. Georgetown University Round Table 2001. Recuperado de http://www.ling.upenn.edu/ wlabov/uesn.pdf

Labov, W. (2011). Oral narratives of personal experience. Cambridge: Cambridge University Press.

Leonard, L. (2014). Children with specific language impairment. Massachusetts: The MIT Press.

Mendoza, E. (Coord.). (2014). Trastorno Específico del Lenguaje (TEL). Madrid: Ediciones Pirámide.

Morgan, G., Restrepo, A., \& Auza, A. (2009). Variability in the grammatical profile of Spanish-speaking children with specific language impairment. En J. Grinstead (Ed.), Hispanic Child Languages 50 (pp. 283-309). http://dx.doi.org/10.1075/lald.50.13mor
Pavez, M. M., Coloma, C. J., y Maggiolo, M. (2008). EI desarrollo narrativo en niños. Barcelona: Ars Médica.

Pérez, E. (1997). Cohesión y coherencia en las narraciones de niños y niñas con Trastorno Específico del Lenguaje. Revista de Logopedia, Foniatría y Audiología, 17(2), 103111. http://dx.doi.org/10.1016/s0214-4603(97)75658-4

Serra, M. (1997). Dificultades cognitivas y lingüísticas en los niños con trastorno específico del lenguaje. Revista de Logopedia, Foniatría y Audiología, 17(2), 79-91. http://dx.doi.org/10.1016/s0214-4603(97)75656-0

Shiro, M. (2007). La construcción del punto de vista en los relatos orales de niños en edad escolar: un análisis discursivo de la modalidad. Venezuela: Fondo Editorial de Humanidades y Educación.

Vázquez, P. (2013). Plano morfosintáctico. En B. Aizpún et al., (Eds.), Enfoque neurolingüístico en los trastornos del lenguaje infantil. Buenos Aires: Editorial Akadia.

\footnotetext{
Agradecimientos

Quisiera agradecer a la profesora adjunta Licenciada en Fonoaudiología Carmen Caligari por permitirme asistir y grabar sus sesiones en el Hospital Pereira Rossell y a la Directora de la carrera de Fonoaudiología de la EUTM, Facultad de Medicina de la Universidad de La República, la Profesora Agregada Alicia Munyo
} 


\section{Anexo}

\section{Corpus 1}

$R: Y$ camién te tengo que contar algo más.

F: A ver contame...

R: Sí... Bueno... jah! sí, te teee nemos un nuevo de la familia, que camién es de la familia.

F: A ver a ver que eso se está poniendo muy interesante...

R: Aier vino.

F: ¿Quién vino?

R: ¡Un bebé! Mamá quería un perrito raza, raza, raza eh... viste esos pelos que camién tienen rulos que camién.

F: Caniche.

R: Mamá tuvo porque lo sacó de la panza de la made porque (XXX).

F: ¿Porque qué?

R: Mamá lo compró.

F: ¿Dónde lo compró mamá al caniche toy?

R: No lo sé pero lo compro, bueno io no sabía... Bueno en pimera clase sabía que camién iba a compar al caniche toy pero no sabía que camién ia aier... Así que aier, aier me ievó a la escuela no sé qué hizo que camién vine de la escuela, salí porque no sé qué hice porque estuve en la escuela.

F: ¿Y tenés que saber lo que hizo mamá mientras vos estás en la escuela?

R: iNo!

$\mathrm{F}:\lceil\mathrm{Ah}$ !

R: Pero no sé lo que hizo así que no me pegunten, así que salí de la escuela, mamá estaba con la mochila abierta, la mochila así ( $R$ hace gesto y cara de sorpresa, como si la mamá le mostrase algo que tiene dentro de su mochila); entonces me dijo "vení", me ievó del brazo y había un XXX.

F: ¿Había un qué?

R: Lo vamos en el taxi, mamá me ievó de la mano y nos vamos al taxi. Porque mamá buscó al taxi.

F: ¡De apuro te llevó rapidito!

R: Entonces vi al perro, XXX con los ojos cerados y listo. Lo vi, mamá lo bajamos del taxi, fuimos pa casa luego tenía deberes igracias a Dios! Porque cooon es que como que camién mi maesta particular se fue el martes.

F: ¿Vas a maestra particular?

R: Sí, voy a maesta paticular pero ahora que camién aier hacen turnos. Uun como que un día es que hahaago deberes con mami y camién otos días con mamá. Se turnan.

F: No vas todos los días a las clases con la maestra.

R: ¿Eh? 
F: ¿No vas todos los días a clases con la maestra particular?

$R$ : No porque cooomo que eia se fue el martes afuera y camién viene el lunes.

F: $R$, a ver si te entendí bien... Tú no hacés todos los días los deberes con mamá porque hay días que los hacés con la maestra y otros días con mamá. ¿Eso es lo que me quisiste contar?

R: No, que casi todo toodo los días lo hago con la maesta paticular para eso lo pagan, pero un día, el martes cooomo como que se fue de afuera, me dijo, se fue de afuera, que camién viene el lunes camién eso pasó el martes.

I: R, yo no entendí lo del perrito... Mamá te sacó volando en un taxi. Y ese taxi ¿a dónde las llevó?

R: Al barrio sobe mi casa.

I: Al barrio de tu casa, ¿Y qué hicieron cuando llegaron al barrio de tu casa?

R: Caminamos y camién fuimos a casa.

I: ¿Y el perrito?

R: Estaba dumiendo pero camién luego lo sacamos, mamá le compó tarros, y camién pasó la noche un poco iorando.

I: Y claro, decime con tus manitos cómo es de tamaño (Romina muestra), ies chiquito! ¡Debe extrañar a la mamá!

R: Sí y camién deee noche como que iora un poquito hasta de noche tuvimos que cuidalo.

I: Claro, los cachorros dan trabajo.

R: ¡No! No era por el cabajo, era por un pobema más maior.

I: ¿Qué pasó?

R: El pito.

I: ¿Qué le pasó en el pito?

R: El Pito es un gato.

(RISAS)

R: Entonces el perrito se llama Lucho.

C: ¿Y cuál fue ese gran problema de la noche?

R: Bueno, a pimera ela con el gato malo que camién es el Pitu, más mañoso y el gato más y más y más quiaro por casa que he visto que camién crió en casa, se fue quedando en casa, está atado porque vive en casa.

I: Pero ¿qué pasó con el gato cuando llegó el perrito?

R: El Pitu nooo se qué pasó pero no quiere a male y cuando male se acerca se aleja de él. Eso lo que no entiendo Pero camién el Pitu araña a los perros.

I: ¿Y lo arañó a Lucho?

R: Po suerte no pero (XXX) Entonces le pegué. Nunca quiere a los perros, es mañoso.

I: Pero y Lucho lloraba porque se daba cuenta que el gato... 
R: No, no, no. Lucho ioraba siempe. Es bebé.

I: Entonces, la historia del taxi es así: mamá $\mathrm{f}$

R: Sí pero camién el perro no le hizo nada.

$(\ldots)$

I: A ver si entendí. Ayer tu mamá te fue a buscar a la escuela, te sacó corriendo, te subió en un taxi. Se fueron juntas a tu casa porque quería mostrarte a Lucho como sorpresa. ¿Vos viste a Lucho por primera vez cuando llegaste de la escuela?

R: Asiente con la cabeza.

I: ¡Ah! ¡Porque no había entendido!

\section{Cuento “Óscar tiene frío" de Ricardo Alcántara}

\section{Presentación}
a) Marco
b) Ubicación espacial y temporal
c) Problema

Los osos son tan grandes que les cabe mucho sueño en el cuerpo, por eso pueden dormir durante todo el invierno.

Precisamente, eso fue lo que hizo Óscar: dormir y dormir a pata suelta. Pasó el invierno encerrado en su casa, sin más compañía que sus ronquidos y sus sueños.

Pero, tan pronto llegó la primavera, abrió los ojos y comenzó a desperezarse.

-iAh! iMmmm! iUuuh! - exclamaba el oso, mientras estiraba un brazo, una pata o balanceaba la cabeza.

Luego, sin prisas, se acercó a la ventana y paseó la vista por el paisaje.

-iQué bien! iHa llegado la primavera! - celebró muy contento.

En efecto, solo se veían restos de nieve a lo lejos, las hojas ya verdeaban en las ramas de los árboles y el sol brillaba con ganas.

Sin embargo, a pesar del buen tiempo, Óscar sentía un frío que le recorría todo el cuerpo y no lo dejaba estar tranquilo.

\section{Episodio 1}
a) Meta
b) Intento para alcanzar la meta
c) Consecuencia

"Quizá andando consiga entrar en calor", se dijo el oso, y se puso a caminar. 
¡Caminó y caminó y caminó, pero inada!

—iQué te pasa? - le preguntó la liebre al ver que le castañeteaban los dientes.

-Tengo mucho frío- respondió el oso.

-No te preocupes, eso lo arreglo yo- dijo la liebre y salió disparada.

Regresó al cabo de un rato con una manta de lana muy gruesa y abrigada.

-Envuélvete con ella y verás cómo el frío deja de molestarte.

Óscar así lo hizo. Se echó la manta sobre los hombros y continuó su camino.

Caminó y caminó y caminó envuelto en la manta, pero inada! El frío no dejaba de soplarle con su aliento helado.

\section{Episodio 2}
a) Meta
b) Intento para alcanzar la meta
c) Consecuencia

—¿Qué te pasa? - le preguntó la urraca al verlo tiritando.

-Tengo... tengo frío- respondió Óscar.

- Conozco un buen remedio para tus males. No te muevas de aquí, ahora mismo te lo traigo dijo la urraca, y salió volando.

Regresó poco después con una tableta de chocolate.

—Cómelo de a poco. Ya verás, ite sentirás muy bien!

-Gracias- dijo Óscar, y siguió su camino.

Caminó y caminó y caminó envuelto en la manta y comiendo chocolate, pero inada! El frío continuaba acariciándole con sus manos de escarcha.

\section{Episodio 3}
a) Meta
b) Intento para alcanzar la meta
c) Consecuencia

—¿Qué te pasa? - le preguntó el zorro al verle pálido, con los pelos de punta y la piel de gallina.

-Tengo mucho frío - respondió el oso.

-Eso se soluciona fácilmente- dijo el zorro, y salió corriendo.

Regresó casi al momento con leña. 
En un abrir y cerrar de ojos preparó una hoguera.

-Siéntate junto al fuego. Ya verás, rápidamente entrarás en calor.

Óscar se sentó junto al fuego, con la manta sobre los hombros y dándole mordiscos al chocolate, pero inada! No había manera de sacarse el frío del cuerpo. 